\title{
Assessment of the degree of hydrological indicators alteration under climate change
}

\author{
Chunxue $\mathrm{Yu}^{1,2, a}$, Xin'an Yin ${ }^{3, b}$, Zhifeng Yang ${ }^{2,3, c}$ and Zhi Dang ${ }^{1, d}$ \\ ${ }^{1}$ School of Environmental Science and Engineering, South China University of Technology, \\ University Town, Guangzhou, China \\ ${ }^{2}$ Research Center for Eco-Environmental Engineering, Dongguan University of Technology, \\ Dongguan, China \\ ${ }^{3}$ State Key Laboratory of Water Environmental Simulation, School of Environment, Beijing Normal \\ University, Beijing, China

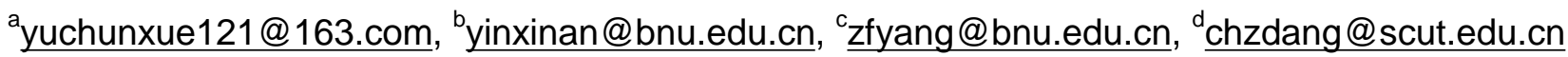

Keywords: Reservoir storage; Ecological requirement; Optimization model; Climate change. Abstract. The native biodiversity and integrity of riverine ecosystems are dependent on the natural flow regime. Maintaining the natural variability of flow in regulated river is the most important principle for the operation and management of environmental flow (e-flow). However, climate change has altered the natural flow regime of rivers. Flow regime alteration is regarded as one major cause leading to the degradation of riverine ecosystems. It is necessary to incorporate the impacts of climate changes into e-flow management. To provide scientific target for e-flow management, the assessment method for flow regime alteration is developed. We analyze the alteration of hydrological indicator under climate change. This study has selected the commonly used Indicators of Hydrologic Alteration (IHAs) to describe the various aspects of flow regimes. To assess the alteration degree of each IHA in regulated river under climate change, GCM is used to generate feasible future climate conditions and hydrological model is used generate flow of river from those future weather conditions. Then the values of IHAs are derived from historical and simulated flow data. The relationship between IHA and climate variable is analyzed based on analysis method. The IHA, which is more influenced by the climate variables, can be selected for the preparation of reservoir operation.

\section{Introduction}

Analysis of the alteration of natural flow regime is based on appropriate hydrological indicators to quantitatively describe the characteristics of river inflow at different time scales. Now, there are nearly 170 hydrological indicators which have been used to assess flow regime alteration [1]. Early the hydrological deviation method for flow regime alteration assessment is mainly to reflect the difference between the measured flow rate and the natural flow. The method contains too little hydrological information and basically does not reflect the ecological information. Then with the continuous development of ecological hydrology, a large number of eco-hydrology indicators have been developed. Flow-duration curves (FDC) are widely used in flow regime alteration assessment and it could directly reflect the alteration [2]. Based on FDC, Homa et al [3] introduce introduces the concept of 'Ecodeficit' and 'Ecosurplus' to evaluate the flow regime alteration at different time scales and the related ecological impacts. These methods are simple and easy to operate, but they cannot fully reflect all five aspects of flow regime (magnitude, duration, timing, frequency and rate of change). To solve this problem, Richter et al [4] established a set of Indicators of Hydrologic Alteration (IHA) to assess the flow regime alteration before and after interference of climate change and human activity. Currently, IHA is the most widely used method in the world [5, 6]. The 32 IHAs are categorized by five groups of hydrological features, that is, flow magnitude, duration, timing, frequency and rate of change.

Climate change is one of the main drivers of flow regime alteration in the river and it is affecting the rivers around the world [7,8]. Due to human activity, increasing greenhouse gas emissions will lead to global warming [9]. The rise in temperature will inevitably lead to an increase in the rate of 
snow melting and evapotranspiration, and finally change the flow regime [10]. At present, the change of the natural flow regime induced by climate change has aroused more and more extensive attentions. Scholars in the related fields all over the world have carried out a lot of research to assess the changes of hydrological indicators in different future climate scenarios and their effects on the river ecosystem.

In this study, a group of commonly used indicators (Indicators of Hydrologic Alteration, IHA) were selected to explore the impact of climate change on flow regime. First, the large-scale climatic factors (from the climate simulation model) were downscaled to low-resolution and regional scale climatological factor data. The regional climatic factor data were imported into the hydrological model to obtain inflow for the study area. The multiple technologies are further used to explore the changes in hydrological indicators in different climate scenarios. The overall target of this study is to quantify the value of ecological hydrological indicators and their changes in climate change.

\section{Methodology}

Weather generator module. This study selected a typical global climate model ECHAM as the atmospheric circulation model (GCM) to generate basic information on large-scale climate predictors in future climate scenarios. The detail information of ECHAM can be seen in Table 1. Emission scenarios A2 (high greenhouse gas emission scenarios) and B1 (low greenhouse gas emission scenarios) are selected.

Table 1 The basic information of the ECHAM model

\begin{tabular}{llllll}
\hline Model & Country & Atmospheric resolution & Marine resolution & Range & Grid-point \\
\hline ECHAM & Germany & $1.875^{\circ} \times 1.875^{\circ}$ & $1^{\circ} \times 1^{\circ}$ & $89^{\circ} \mathrm{N}-89^{\circ} \mathrm{S}$ & $144 \times 90$ \\
& & & $1.25^{\circ} \mathrm{E}-358.75^{\circ} \mathrm{E}$ & \\
\hline
\end{tabular}

Table 2 lists the variables in the global climate model (ie, candidate forecast factors). In this study, the daily rainfall, maximum temperature and minimum temperature were chosen as the forecasting factors. The future meteorological factor prediction is achieved by the above-established statistical relationship and the large-scale climate prediction factor generated by the ECHAM climate model. There are some small differences in the statistical reduction of rainfall, maximum temperature and minimum temperature.

Table 2 GCMs large-scale alternative factors

\begin{tabular}{lll}
\hline Variable & Long Name & Stardard Name \\
\hline hur & Relative Humidity & Relative_humidity \\
hus & Specific Humidity & specific_humaidity \\
huss & Surface Specific Humidity & specific_humaidity \\
pr & Precipitation & Precipitation_flux \\
psl & Sea Level Pressure & air_pressure_at_sea_level \\
ta & Temperature & air_temperature \\
tas & Surface air temperature & air_temperature \\
tasmax & Maximum daily surface air temperature & air_temperature \\
tasmin & Minumum Daily surface air Temperature & air_temperature \\
zg & Geopotential Height & Geopotential_height \\
\hline
\end{tabular}

The statistical downscaling model is a decision support tool based on the Windows interface, research area and local climate change impact established by Wilby et al [11]. The model combines the weather generator and multiple linear regression techniques, which is a hybrid statistical descent method and is widely used in related climate change research.

Hydrologic simulation module. Compared with other hydrological models, distributed hydrological models are often used for runoff forecasting. VMOD, because of its open source, with a certain physical meaning and parameterization process relatively clear, is widely used in the world. At 
present, it is possible to simulate the impact of climate change on hydrological cycles and runoff output [12]. So in this study we use the VMOD hydrological model to do runoff simulation. The basic data of the research area to be established in this study are obtained through field survey, site collection and literature review.

Assessment of the IHA's alteration. The IHAs used in this paper are shown in Table 3. The 32 IHAs are categorized by five groups of hydrological features, that is, flow magnitude, duration, timing, frequency and rate of change. Based on the value of the 32 IHAs under the base period and climate change condition, we carry out to analyze the impact of climate change on river hydrological indicators.

Statistical analysis: In order to test the degree of change in the indicators, the change degree of the indicators can be calculated by the mean or median of the IHA. The variability of the indicators can be measured using the IHA standard deviation or the number of bits between the $25^{\text {th }}$ and $75^{\text {th }}$ quartiles. This study selects the median and the number of divisions between the $25^{\text {th }}$ and $75^{\text {th }}$ quartiles to measure the degree of change degree of IHA.

Table 3 Indicators of hydrological alteration (IHA) in the range of variability approach

\begin{tabular}{|c|c|c|}
\hline IHA group & Hydrological indicators & Abbreviation \\
\hline $\begin{array}{l}\text { Group 1: Magnitude of } \\
\text { monthly water conditions }\end{array}$ & Mean value for each calendar month & $\begin{array}{l}\text { JANF, FEBF, MARF, APRF, MAYF, } \\
\text { JUNF, JULF, AUGF, SEPF, OCT, } \\
\text { NOVF, DECF }\end{array}$ \\
\hline \multirow{10}{*}{$\begin{array}{l}\text { Group 2: Magnitude and } \\
\text { duration of annual extreme } \\
\text { water conditions }\end{array}$} & Annual minima 1-day means & MI1F \\
\hline & Annual maxima 1-day means & MA1F \\
\hline & Annual minima 3-day means & MI3F \\
\hline & Annual maxima 3-day means & MA3F \\
\hline & Annual minima 7-day means & MI7F \\
\hline & Annual maxima 7-day means & MA7F \\
\hline & Annual minima 30-day means & MI30F \\
\hline & Annual maxima 30-day means & MA30F \\
\hline & Annual minima 90-day means & MI90F \\
\hline & Annual maxima 90-day means & MA90F \\
\hline \multirow{7}{*}{$\begin{array}{l}\text { Group 3: Timing of annual } \\
\text { extreme water conditions } \\
\text { Group 4: Frequency and } \\
\text { duration of high and low } \\
\text { pulses }\end{array}$} & Julian date of each annual 1 day maximum & TMIM \\
\hline & Julian date of each annual 1 day minimum & ТМАМ \\
\hline & No. of high pulses each year & NHP \\
\hline & No. of low pulses each year & NLP \\
\hline & Mean duration of high pulses within each & DHP \\
\hline & & DLP \\
\hline & $\begin{array}{l}\text { Mean duration of low pulses within each } \\
\text { year }\end{array}$ & \\
\hline \multirow[t]{3}{*}{$\begin{array}{l}\text { Group 5: Rate and frequency } \\
\text { of water condition changes }\end{array}$} & $\begin{array}{l}\text { Means of all positive differences between } \\
\text { consecutive daily means }\end{array}$ & MPD \\
\hline & $\begin{array}{l}\text { Means of all negative differences between } \\
\text { consecutive daily values }\end{array}$ & MND \\
\hline & No. of exchange & NREV \\
\hline
\end{tabular}

\section{Study area}

Taizi River Basin (longitude $122^{\circ} 30^{\prime}-124^{\circ} 50^{\prime}$; latitude $40^{\circ} 30^{\prime}-41^{\circ} 40^{\prime}$ ) is located in the eastern part of Liaoning Province, and the basin area is $1.4 \times 10^{4} \mathrm{~km}^{2}$. It belongs to the mid-latitude region, affected by climate change is more significant. The total length is $413 \mathrm{~km}$. Taizi River Basin is the industrial and agricultural production base of Liaoning Province, which has important research value. 


\section{Results and discussion}

Future climate character analysis. Based on NECP and ECHAM, the daily rainfall, minimum temperature and maximum temperature data of Liaoyang station in Taizi River reference period (1900-1959) and future (2011-2001) were obtained by downscaling. The results of the data analysis of the precipitation are shown in Figures 1. Other two factors (minimum temperature and maximum temperature) have the same change trends and we will not list in this study.

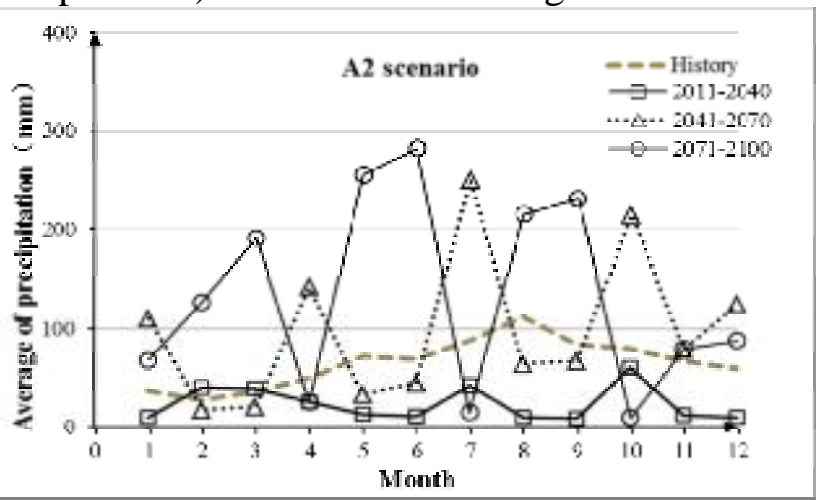

(a)

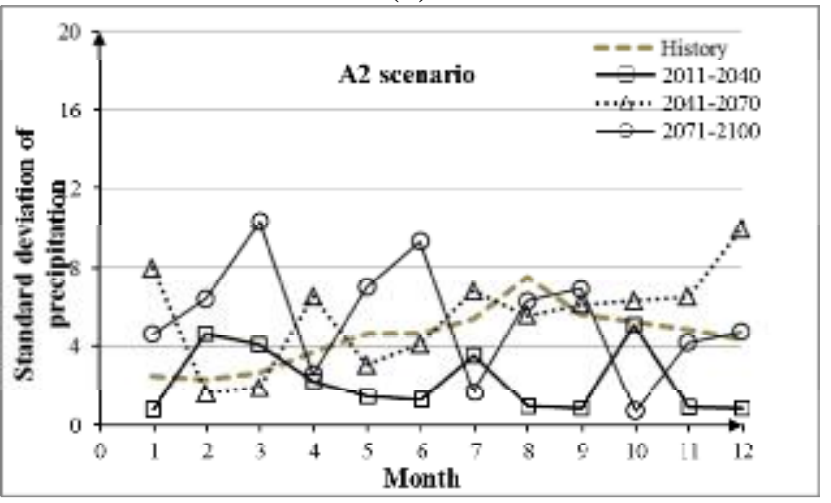

(c)

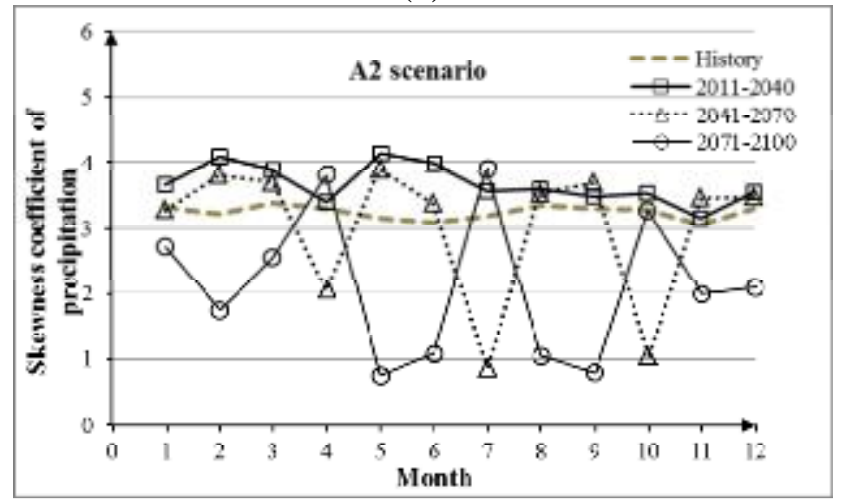

(e)

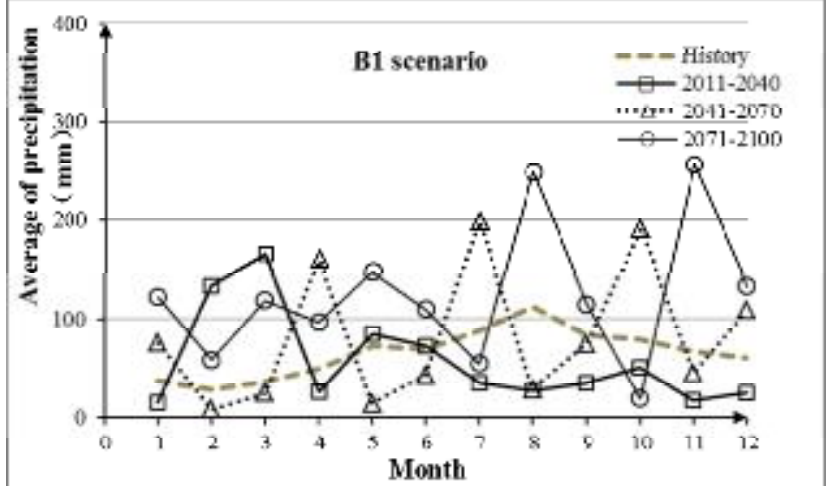

(b)

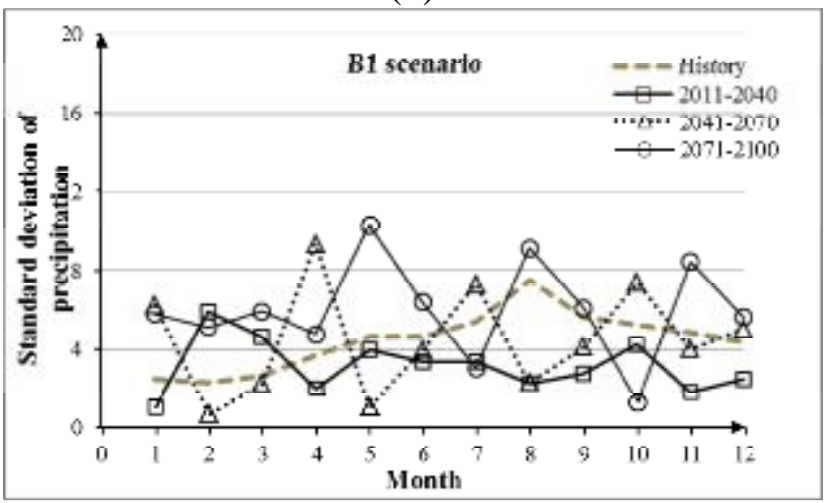

(d)

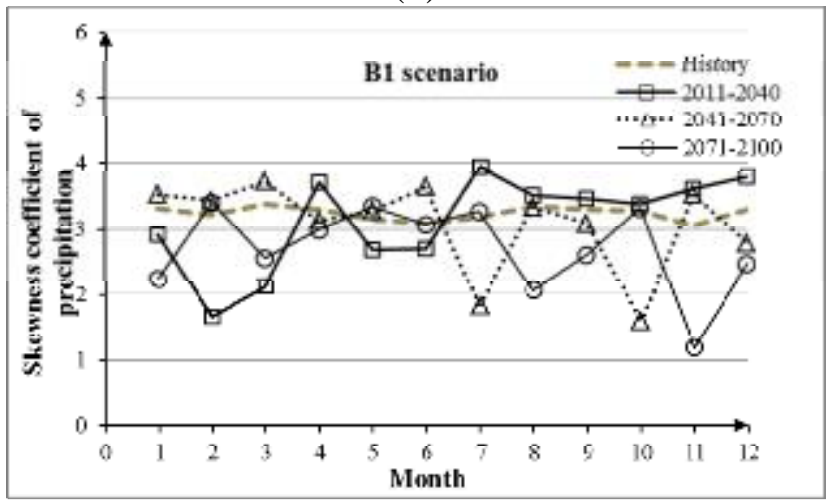

(f)

Figure 1 Projected changes in precipitation of each month at Liaoyang station in the future periods under different climate scenarios

It can be seen from Figure 1 that, in the A2 scenario ( high greenhouse gas emission) or in the B1 scenario (low greenhouse gas emissions), compared with the precipitation in the reference period 1900-1959, the precipitation in the Liaoyang station has changed from three periods of time 2011-2040, 2041-2070 and 2071-2001. The range of variation is $-90 \% \sim 425 \%$. In the A2 scenario, the average monthly precipitation in Liaoyang station was lower than the mean value during the period of 2011-2040; 2041 - 2070 and 2071-2100 were drastically changed compared with the base period. It can be seen that the difference between the months of the A2 scenario is large. In the case of B1 scenario, the mean change is smaller than that of the A2 scenario, $-80 \%$ to $369 \%$. Figure 1 (c) and (d) describe the monthly standard deviation of precipitation $(\mathrm{Cv})$ for the next three periods in both $\mathrm{A} 2$ and B1 scenarios. The magnitude of the change is relatively large, indicating that the deviation from 
the base period of the degree of large. Figure 1 (e) and (f) describe the skewness coefficient (Cs) of precipitation, which shows that all scenarios show a positive partial distribution (Cs>0). The skewness coefficient is greater than 0 , because the mean is in the right of the public. It is a right partial distribution, also known as positive bias.

Table 4 IHA values for the reference period 1900-1959 and projected future changes in IHA for the period 2011-2040, 2041-2070 and 2071-2100.

\begin{tabular}{|c|c|c|c|c|c|c|c|}
\hline \multirow{2}{*}{ IHA } & \multirow{2}{*}{$\begin{array}{c}1900- \\
1959\end{array}$} & \multicolumn{2}{|l|}{ 2011-2040 } & \multicolumn{2}{|l|}{ 2041-2070 } & \multicolumn{2}{|c|}{ 2071-2100 } \\
\hline & & A2 & B1 & $\mathbf{A 2}$ & B1 & $\mathbf{A 2}$ & B1 \\
\hline \multicolumn{8}{|c|}{ Group 1: Magnitude of monthly water conditions } \\
\hline JANF & 30.9 & $+0.5 \uparrow 1.8 \%$ & $-2.4 \downarrow 7.8 \%$ & $-3.0 \downarrow 9.8 \%$ & $-4.7 \% \downarrow 15.4 \%$ & $-7.4 \downarrow 24.1 \%$ & $-4.2 \downarrow 13.7 \%$ \\
\hline FEBF & 24.2 & $+3.5 \uparrow 14.3 \%$ & $+2.7 \uparrow 11.4 \%$ & $+2.6 \underset{\%}{\uparrow 10.8}$ & $+2.8 \uparrow 11.5 \%$ & $+2.6 \uparrow 10.7 \%$ & $+3.3 \uparrow 13.6 \%$ \\
\hline MARF & 35.7 & $+5.0 \uparrow 13.9 \%$ & $+2.2 \uparrow 6.2 \%$ & $+1.8 \uparrow 5.2 \%$ & $+3.0 \uparrow 8.4 \%$ & $-6.0 \downarrow 16.8 \%$ & $-7.3 \downarrow 20.6 \%$ \\
\hline APRF & 77.1 & $-37.7 \downarrow 49.0 \%$ & $-44.8 \downarrow 58.1 \%$ & $-44.1 \downarrow 57.2 \%$ & $-42.9 \downarrow 55.6 \%$ & $-48.2 \downarrow 62.4 \%$ & $-47.4 \downarrow 61.5 \%$ \\
\hline MAYF & 55.2 & $-18.9 \downarrow 34.2 \%$ & $-30.8 \downarrow 55.7 \%$ & $-28.7 \downarrow 52.0 \%$ & $-28.9 \downarrow 52.4 \%$ & $-27.3 \downarrow 49.4 \%$ & $-31.7 \downarrow 57.4 \%$ \\
\hline JUNF & 47.8 & $-10.4 \downarrow 21.7 \%$ & $-19.8 \downarrow 41.4 \%$ & $-5.8 \downarrow 12.1 \%$ & $-16.6 \downarrow 34.7 \%$ & $-16.2 \downarrow 33.9 \%$ & $-20 \downarrow 41.8 \%$ \\
\hline JULF & 146.0 & $+92.5 \uparrow 63.4 \%$ & $-20.0 \downarrow 13.7 \%$ & $-6.6 \downarrow 4.5 \%$ & $+24.0 \uparrow 16.4 \%$ & $-50.9 \downarrow 34.9 \%$ & $-10.0 \downarrow 6.9 \%$ \\
\hline AUGF & 278.2 & $+40.9 \uparrow 14.7 \%$ & $-62.9 \downarrow 22.6 \%$ & $-76.4 \downarrow 27.4 \%$ & $-74.7 \downarrow 26.8 \%$ & $-109.9 \downarrow 39.5 \%$ & $-79.2 \downarrow 28.5 \%$ \\
\hline SEPF & 137.5 & $-4.6 \downarrow 3.4 \%$ & $-35.7 \downarrow 26.0 \%$ & $-29.2 \downarrow 21.3 \%$ & $-43.9 \downarrow 32.0 \%$ & $-58.3 \downarrow 42.4 \%$ & $-51.0 \downarrow 37.1 \%$ \\
\hline OCTF & 83.5 & $-0.7 \downarrow 1.0 \%$ & $-7.8 \downarrow 9.3 \%$ & $-24.0 \downarrow 28.7 \%$ & $-17.0 \downarrow 20.4 \%$ & $-42.1 \downarrow 50.4 \%$ & $-30 \downarrow 36.0 \%$ \\
\hline NOVF & 61.9 & $-0.9 \downarrow 1.5 \%$ & $-8.2 \downarrow 13.2 \%$ & $-13.8 \downarrow 22.3 \%$ & $-15.6 \downarrow 25.2 \%$ & $-25.5 \downarrow 41.2 \%$ & $-21.6 \downarrow 34.9 \%$ \\
\hline DECF & 41.5 & $+3.5 \uparrow 8.4 \%$ & $-3.1 \downarrow 7.5 \%$ & $-5.1 \downarrow 12.4 \%$ & $-3.0 \downarrow 7.1 \%$ & $-11.5 \downarrow 27.8 \%$ & $-8.6 \downarrow 20.8 \%$ \\
\hline \multicolumn{8}{|c|}{ Group 2: Magnitude and duration of annual extreme water conditions } \\
\hline MI1F & 18.5 & $-0.7 \downarrow 3.9 \%$ & $-3.0 \downarrow 16.2 \%$ & $-3.1 \downarrow 16.8 \%$ & $-3.4 \downarrow 18.2 \%$ & $-4.8 \downarrow 25.7 \%$ & $-3.9 \downarrow 20.8 \%$ \\
\hline MA1F & 1521.8 & $+461.6 \uparrow 30.3 \%$ & $-368.0 \downarrow 24.2 \%$ & $-159.8 \downarrow 10.5 \%$ & $-248.6 \downarrow 16.3 \%$ & $-521.4 \downarrow 34.3 \%$ & $-625 \downarrow 41.1 \%$ \\
\hline MI3F & 55.8 & $-2.0 \downarrow 3.6 \%$ & $-8.1 \downarrow 14.6 \%$ & $-9.2 \downarrow 16.4 \%$ & $-9.2 \downarrow 16.4 \%$ & $-14.0 \downarrow 25.0 \%$ & $-11.1 \downarrow 19.8 \%$ \\
\hline MA3F & 2902.6 & $+1135.3 \uparrow 39.1 \%$ & $-466.9 \downarrow 16.1 \%$ & $-112.3 \downarrow 3.9 \%$ & $-116.4 \downarrow 4.0 \%$ & $-990.5 \downarrow 34.1 \%$ & $-860 \downarrow 29.6 \%$ \\
\hline MI7F & 131.9 & $-4.2 \downarrow 3.2 \%$ & $-18.7 \downarrow 14.2 \%$ & $-20.5 \downarrow 15.6 \%$ & $-17.0 \downarrow 12.9 \%$ & $-31.7 \downarrow 24.0 \%$ & $-25.7 \downarrow 19.5 \%$ \\
\hline MA7F & 4686.4 & $+2188.4 \uparrow 46.7 \%$ & $-862.1 \downarrow 18.4 \%$ & $-273.5 \downarrow 5.8 \%$ & $-49.9 \downarrow 1.1 \%$ & $-1865.2 \downarrow 39.8 \%$ & $-1532 \downarrow 32.7 \%$ \\
\hline MI30F & 641.5 & $-17.1 \downarrow 2.7 \%$ & $-91.8 \downarrow 14.3 \%$ & $-92.1 \downarrow 14.4 \%$ & $-76.7 \downarrow 12.0 \%$ & $-158.1 \downarrow 24.6 \%$ & $-137.4 \downarrow 21.4 \%$ \\
\hline MA30F & 10502.6 & $+4817.1 \uparrow 45.9 \%$ & $-575.1 \downarrow 5.5 \%$ & $-1277.9 \downarrow 12.2 \%$ & $-936.2 \downarrow 8.9 \%$ & $-3911.6 \downarrow 37.2 \%$ & $-2717 \downarrow 25.9 \%$ \\
\hline MI90F & 2676.78 & $+9.5 \uparrow 0.4 \%$ & $-408.8 \downarrow 15.3 \%$ & $-540.4 \downarrow 20.2 \%$ & $-427.1 \downarrow 16.0 \%$ & $-778.5 \downarrow 29.1 \%$ & $-683.1 \downarrow 25.5 \%$ \\
\hline MA90F & 19045.6 & $+4512 \uparrow 23.7 \%$ & $-2575.7 \downarrow 13.5 \%$ & $-566.9 \downarrow 3.0 \%$ & $-4121.3 \downarrow 21.6 \%$ & $-8644.4 \downarrow 45.4 \%$ & $-4873 \downarrow 25.6 \%$ \\
\hline BASF & 1.45 & $+0.03 \uparrow 2.1 \%$ & $+0.2 \uparrow 12.5 \%$ & $-0.01 \downarrow 0.7 \%$ & $-0.1 \downarrow 9.6 \%$ & $+0.5 \uparrow 32.0 \%$ & $+0.06 \uparrow 3.8 \%$ \\
\hline \multicolumn{8}{|c|}{ Group 3: Timing of annual extreme water conditions } \\
\hline TMIM & 75 & $+83.5 \uparrow 111.3 \%$ & $+85.0 \uparrow 113.3 \%$ & $+72 \uparrow 96.0 \%$ & $+76.0 \uparrow 101.3 \%$ & $+68 \uparrow 90.7 \%$ & $+78.5 \uparrow 104.7 \%$ \\
\hline TMAM & 222 & $-5 \downarrow 2.3 \%$ & $-6.5 \downarrow 2.9 \%$ & $-3.5 \downarrow 1.6 \%$ & $-6.0 \downarrow 2.7 \%$ & $-6.5 \downarrow 2.9 \%$ & $-6 \downarrow 2.7 \%$ \\
\hline \multicolumn{8}{|c|}{ Group 4: Frequency and duration of high and low pulses } \\
\hline NHP & 4 & $-1 \downarrow 25 \%$ & $-0.5 \downarrow 12.5 \%$ & $+2 \uparrow 50.0 \%$ & $+1.5 \uparrow 37.5 \%$ & $+1.5 \uparrow 37.5 \%$ & $+2.5 \uparrow 62.5 \%$ \\
\hline NLP & 11 & $-3 \downarrow 27.3 \%$ & $-1.5 \downarrow 13.6 \%$ & $-2 \downarrow 18.2 \%$ & $-4.0 \downarrow 36.4 \%$ & $-4.0 \downarrow 36.4 \%$ & $-3 \downarrow 27.3 \%$ \\
\hline DHP & 71.3 & $+22.0 \uparrow 31.0 \%$ & $+8.8 \uparrow 12.3 \%$ & $-27.1 \downarrow 38.1 \%$ & $-22.4 \downarrow 31.5 \%$ & $-31.0 \downarrow 43.5 \%$ & $-35.7 \downarrow 50.1 \%$ \\
\hline DLP & 25.3 & $+5.8 \uparrow 23.1 \%$ & $+2.9 \uparrow 11.6 \%$ & $+5.9 \uparrow 23.4 \%$ & $+12.3 \uparrow 48.5 \%$ & $+12.2 \uparrow 48.5 \%$ & $+9.7 \uparrow 38.3 \%$ \\
\hline \multicolumn{8}{|c|}{ Group 5: Rate and frequency of water condition changes } \\
\hline MPD & 62.0 & $+15.6 \uparrow 25.2 \%$ & $-13.0 \downarrow 21.0 \%$ & $-3.1 \downarrow 5.0 \%$ & $-18.7 \downarrow 30.1 \%$ & $-32.8 \downarrow 52.9 \%$ & $-26.8 \downarrow 43.3 \%$ \\
\hline MND & 16.76 & $+4.2 \uparrow 25.2 \%$ & $-4.0 \downarrow 24.1 \%$ & $+0.1 \uparrow 0.8 \%$ & $-5.5 \downarrow 33.2 \%$ & $-8.3 \downarrow 49.8 \%$ & $-6.6 \downarrow 39.4 \%$ \\
\hline NREV & 83 & $-2 \downarrow 2.4 \%$ & $-2.0 \downarrow 2.4 \%$ & $+1 \uparrow 1.2 \%$ & $+2.0 \uparrow 2.4 \%$ & $+0.5 \uparrow 0.6 \%$ & $-3 \downarrow 3.6 \%$ \\
\hline
\end{tabular}

Alteration of hydrological indicators. We calculate the all IHA values for the basic period 1900-1959 and future period 2011-2100 under A2 and B1 scenarios, separately. Table 4 shows the change of magnitude and magnitude of the median value of each IHA for three future periods 
(2011-2040, 2041-2070 and 2071-2001). As can be seen from the Table 5, 49 items in the table (cell mark gray) are increasing, which accounts for $25.5 \%$ of the total number. The remaining three quarters of the data is decreasing. It is also can be found that one-third of the growth IHA indicators is under A2 scenario in the 2011-2040 period. Meanwhile, the bold number in the table represent the alteration degree of each IHA in different scenarios. It can be seen that the extreme value under A2 and B1 scenario is during the 2017-2100 period. It indicated that the value of IHA during this period change faster than the mean of historical data.

The variability of IHA is measured by the target range bracketed by the 25 th- and 75 th-percentile values of the target of 32 IHAs. For each IHA indicator, the 25th and 75th percentile of the hydrological disturbance determines the target range of the indicator, and the frequency change of the IHA sequentially can be quantified by comparing the number of years falling within the target range before and after the disturbance. The results are shown in Figure 2. It can be seen from the figure that the change of 18 indicators in A2 scenario is higher than that in B1 scenario, which indicates that the impact of A2 scenario on ecological hydrological indicators is more B2. The change degree of which is TMIM (the date of daily minimum daily flow) is the biggest change, indicating that climate change has a great influence on the date of daily minimum daily flow.

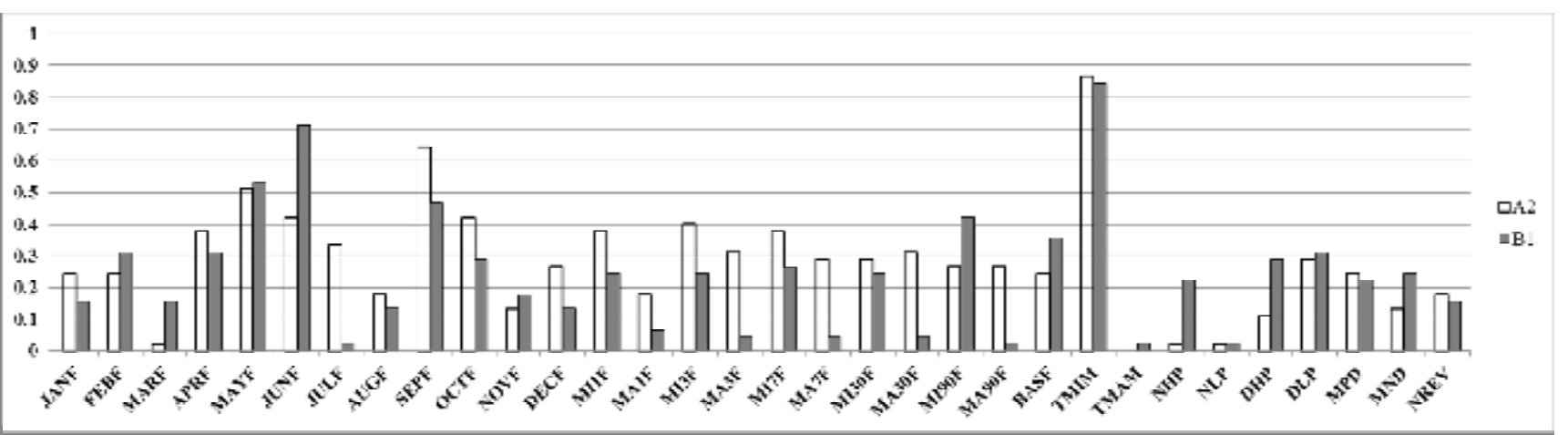

Figure 2 The relationship between different storage ratios and degree of flow regime alteration for various e-flow scenarios

\section{Conclusions}

In this study, the commonly used Indicators of Hydrologic Alteration (IHAs) is adopted to describe the various aspects of flow regime. GCM is used to generate feasible future climate conditions and hydrological model is used generate flow of river from those future weather conditions. Then the value of IHAs are derived from historical and simulated flow data. The alteration of each IHA under different climate change scenarios is determined. The IHA, which is more influenced by the climate variables, can be selected for the preparation of reservoir operation.

\section{Acknowledgements}

This research was supported by the National Key Research Program of China (no. 2017YFC0405903), National Key Research Program of China (no. 2016YFC0502806), National Natural Science Foundation of China (no. 51439001), and the National Science Foundation for Innovative Research Group (no. 51421065).

\section{References}

[1] Olden J D, Naiman R J. Incorporating thermal regimes into environmental flows assessments: modifying dam operations to restore freshwater ecosystem integrity. Freshwater Biology, 2010, 55(1): 86-107. 
[2] Vogel R M, Fennessey N M. Flow-duration curves. I: New interpretation and confidence intervals. Journal of Water Resources Planning and Management, 1994, 120(4): 485-504.

[3] Homa J, Olchawa E, Stürzenbaum S R, et al. Early-phase immunodetection of metallothionein and heat shock proteins in extruded earthworm coelomocytes after dermal exposure to metal ions. Environmental Pollution, 2005, 135(2): 275-280.

[4] Richter B D, Baumgartner J V, Powell J, et al. A method for assessing hydrologic alteration within ecosystems. Conservation Biology, 1996, 10(4): 1163-1174.

[5] Mathews R, Richter B D. Application of the indicators of hydrologic alteration software in environmental flow setting. Journal of the American Water Resources Association, 2007, 43: 1400-1413.

[6] Yu C X, Yin X A, Yang Z F, 2015. A revised range of variability approach for the comprehensive assessment of the alteration of flow regime. Ecological Engineering, 96, 200-207.

[7] Prudhomme C, Davies H. Assessing uncertainties in climate change impact analyses on the river flow regimes in the UK. Part 2: future climate. Climatic Change, 2009, 93(1-2): 197-222.

[8] Döll P, Zhang J. Impact of climate change on freshwater ecosystems: a global-scale analysis of ecologically relevant river flow alterations. Hydrology and Earth System Sciences, 2010, 14(5): 783-799.

[9] Lazzaro, G., Botter, G., 2015. Run-of-river power plants in Alpine regions: Whither optimal capacity?. Water Resources Research, 51(7), 5658-5676. Shakun J D, Clark P U, He F, et al. Global warming preceded by increasing carbon dioxide concentrations during the last deglaciation. Nature, 2012, 484(7392): 49-54.

[10] Barnett T P, Adam J C, Lettenmaier D P. Potential impacts of a warming climate on water availability in snow-dominated regions. Nature, 2005, 438(7066): 303-309.

[11] Wilby R L, Wigley T M L, Conway D, Jones P D, Hewitson B C., Main, J., Wilks D S.,. Statistical downscaling of general circulation model output: a comparison of methods. Water resources research, 1998, 34(11), 2995-3008.

[12] Räsänen T A, Koponen J, Lauri H, Kummu M, 2012. Downstream hydrological impacts of hydropower development in the Upper Mekong Basin. Water Resources Management, 26(12), 3495-3513. 